\title{
Optically detected coherent spin dynamics of a single electron in a quantum dot
}

\author{
M. H. MIKKELSEN, J. BEREZOVSKY, N. G. STOLTZ, L. A. COLDREN AND D. D. AWSCHALOM* \\ Center for Spintronics and Quantum Computation, University of California, Santa Barbara, California 93106, USA \\ *e-mail: awsch@physics.ucsb.edu
}

The ability to sequentially initialize, manipulate and read out the state of a qubit, such as an electron spin in a quantum dot (QD), is a requirement in virtually any scheme for quantum information processing ${ }^{1-3}$. However, previous optical measurements of a single electron spin have focused on time-averaged detection, with the spin being initialized and read out continuously ${ }^{4-8}$. Here, we monitor the coherent evolution of an electron spin in a single QD. We use time-resolved Kerr rotation (KR) spectroscopy, an all-optical, non-destructive technique that enables us to monitor the precession of the spin in a superposition of Zeeman-split sublevels with nanosecond time resolution. The data show an exponential decay of the spin polarization with time, and directly reveal the $g$-factor and spin lifetime of the electron in the QD. Furthermore, the observed spin dynamics provide a sensitive probe of the local nuclear spin environment.

The magneto-optical Kerr effect results in a rotation of the plane of polarization of linearly polarized light on reflection off the sample, analogous to the Faraday effect for transmitted light. For a probe laser energy $E$, the $\mathrm{KR}$ angle, $\theta_{\mathrm{K}}$, is proportional to the difference of the dynamic dielectric response functions for left and right $\left(\sigma^{+}\right.$and $\left.\sigma^{-}\right)$circularly polarized light ${ }^{9}$, which are proportional to the modulus squared of the interband momentum matrix elements $P_{c, v}^{\sigma^{ \pm}}=\left\langle\psi_{\mathrm{c}}\left|\hat{p}_{x} \pm i \hat{p}_{y}\right| \psi_{\mathrm{v}}\right\rangle$, where $\left|\psi_{\mathrm{c}}\right\rangle\left(\left|\psi_{\mathrm{v}}\right\rangle\right)$ is a conduction (valence) band state and $\hat{p}_{x, y}$ are momentum operators. For a single conduction band energy level in a QD containing a spin-up electron in a state $\left|\psi_{\uparrow}\right\rangle$, optical transitions to the spin-up state are forbidden by the Pauli exclusion principle. Considering only the transitions from a single twofold-degenerate valence-band level $\left|\psi_{\mathrm{v}_{0}}\right\rangle, \theta_{\mathrm{K}}$ is given by

$$
\theta_{\mathrm{K}}(E)=C E\left(\left|P_{\downarrow, \mathrm{v}_{0}}^{\sigma^{+}}\right|^{2}-\left|P_{\downarrow, \mathrm{v}_{0}}^{\sigma^{-}}\right|^{2}\right) \frac{E-E_{0, \mathrm{v}_{0}}}{\left(E-E_{0, \mathrm{v}_{0}}\right)^{2}+\Gamma_{0, \mathrm{v}_{0}}^{2}},
$$

where $E_{0, v_{0}}$ is the energy of the transition, $\Gamma_{0, v_{0}}$ is the linewidth of the transition and $C$ is a material-dependent constant. In a QD containing a single conduction-band electron, the lowest-energy interband transition is to the negatively charged exciton state, $\mathrm{X}^{-}$, with an energy $E_{\mathrm{X}^{-}}$. Thus, as has recently been demonstrated ${ }^{4,5}$, a single electron spin produces a feature in the KR spectrum with the odd-lorentzian lineshape given by equation (1), centred at the energy $E_{\mathrm{X}^{-}}$.

In the present work, the electron is confined to a single QD formed by monolayer fluctuations at the interfaces of a gallium arsenide (GaAs) quantum well. The QD is embedded within a diode structure, enabling controllable charging of the dot with a bias voltage ${ }^{10}$. Also, the QD is centred within an integrated optical cavity to enhance the small, single-spin KR signal ${ }^{4}$. With
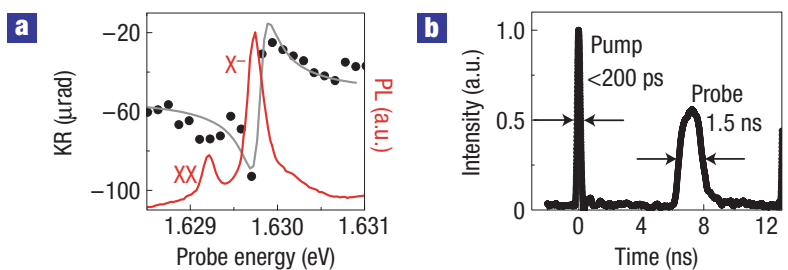

C

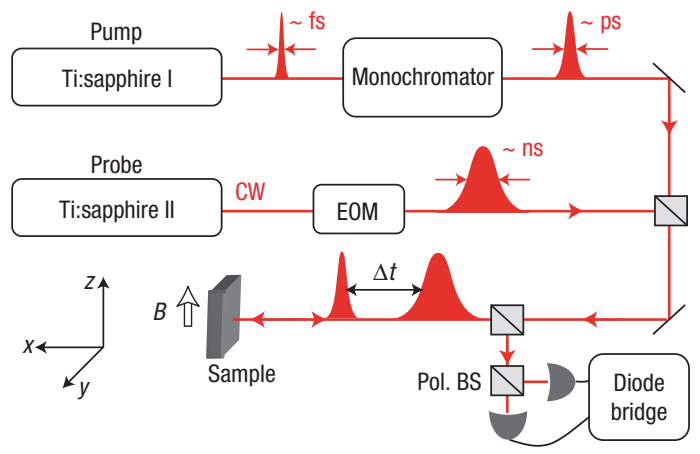

Figure 1 Experimental set-up for single-spin detection. $a, X^{-}$and biexciton $(X X)$ $P L$ lines from $a \mathrm{QD}$ and the corresponding KR spectrum measured with continuous-wave pump and probe, $B=0 \mathrm{G}$. The grey line is an odd-lorentzian fit centred at the $X^{-}$energy, demonstrating single-electron spin detection. $\mathbf{b}$, The profiles of the pump and probe pulses as detected with a photodiode. The measured width of the pump pulse is limited by the $2 \mathrm{GHz}$ bandwidth of the photodiode. c, Schematic diagram of the experimental set-up (EOM, electro-optic modulator; Pol. $\mathrm{BS}$, polarizing beam-splitter).

circularly polarized excitation, spin-polarized electrons and holes are pumped into the quantum well, according to the selection rules governing interband transitions in GaAs (ref. 11). One or more electrons and/or holes then relax into the QD. By measuring the subsequent single-QD photoluminescence (PL), we determine the equilibrium charge state of the QD as well as the energies of various interband optical transitions as a function of bias voltage $e^{4,12}$. The measurements described below are made at a bias voltage where the QD is nominally uncharged, and the optical excitation injects one or more electrons or holes. In this regime, the QD may contain a single spin-polarized electron through the capture of an optically injected electron, or spin-dependent $\mathrm{X}^{-}$decay $^{4}$. Knowing the transition energy $E_{\mathrm{X}^{-}}$from the PL measurements, we use the spectroscopic dependence of the Kerr effect to isolate the dynamics 

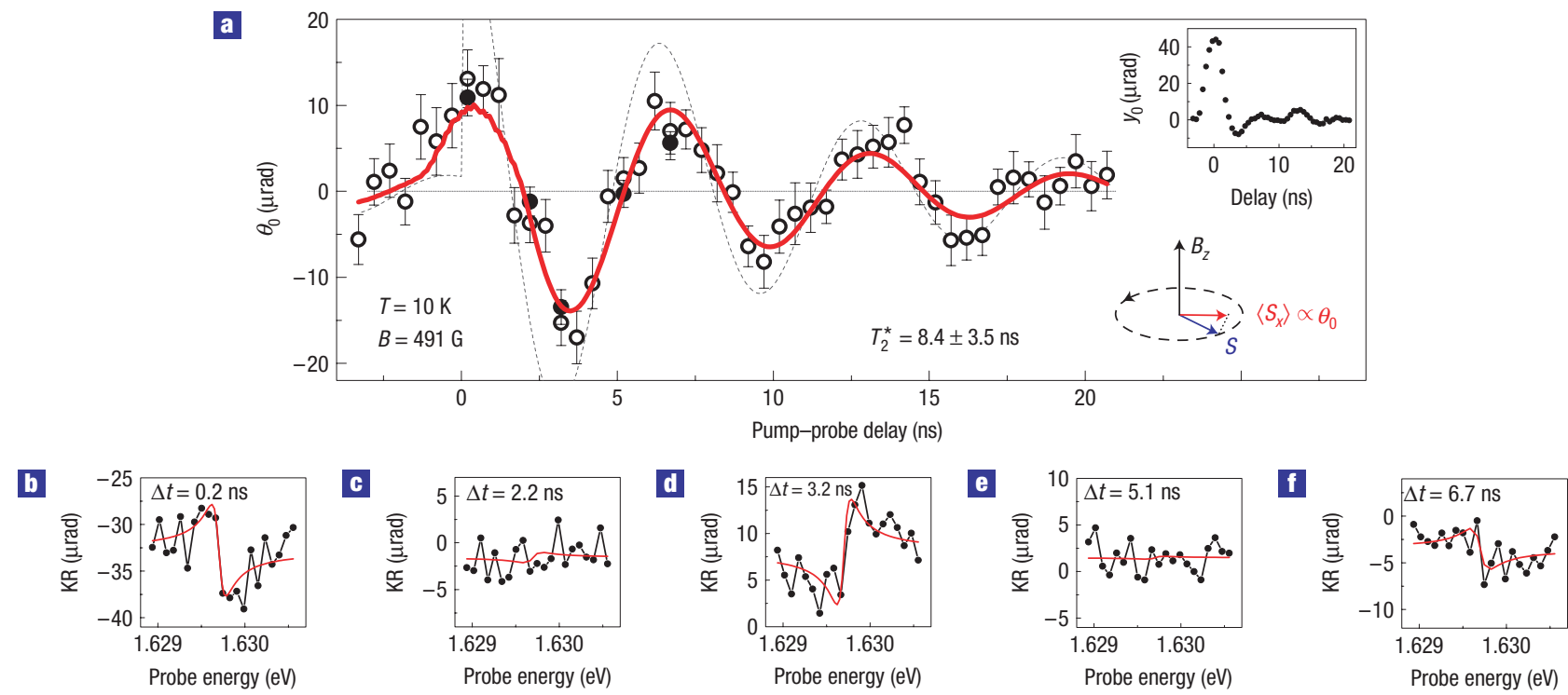

Figure 2 Coherent evolution of a single electron spin. a, Single-spin KR amplitude, $\theta_{0}$, as a function of time, with 3-ns-duration probe pulses and $B=491 \mathrm{G}$. The solid line is a fit to equation (2) and the dashed line shows the solution of equation (2) without the probe-pulse convolution for the same fit parameters. The inset shows the offset, $y_{0}$. Error bars indicate the standard error as obtained from the least-squares fit to the KR spectra. The solid circles indicate the values of $\theta_{0}$ obtained from the fits shown in $\mathbf{b}-\mathbf{f}$. b-f, KR angle as a function of probe energy at five different delays; solid lines are fits to equation (1), with a constant offset, $y_{0}$.

of the single electron spin from that of multiparticle complexes, such as charged or neutral excitons. Figure la shows the $\mathrm{X}^{-}$and biexciton PL lines from a QD, along with the corresponding KR spectrum measured with a continuous-wave pump and probe. The odd-lorentzian feature centred at the $\mathrm{X}^{-}$energy demonstrates the time-averaged measurement of a single electron spin.

In previous work, only the steady-state spin polarization was measured, concealing information about the evolution of the spin state in time. Here, we use time-delayed pump and probe pulses, shown schematically in Fig. 1c, to map out the coherent dynamics of the spin in the QD. The pump and probe pulses, as detected by a fast photodiode, are shown in Fig. 1b. Details of the experimental set-up are given in the Methods section.

For a fixed delay between the pump and the probe, $\theta_{\mathrm{K}}$ is measured as a function of probe energy. At each point, the pump excitation is switched between $\sigma^{+}$and $\sigma^{-}$polarization at a rate $1 / t_{\text {switch }}$, and the spin-dependent signal is obtained from the difference in $\theta_{\mathrm{K}}$ at the two helicities. The resulting KR spectrum is fitted to equation (1) plus a constant vertical offset, $y_{0}$. The amplitude, $\theta_{0}=C E\left(\left|P_{\downarrow, v_{0}}^{\sigma^{+}}\right|^{2}-\left|P_{\downarrow, v_{0}}^{\sigma^{-}}\right|^{2}\right) \Gamma_{0, v_{0}}^{-1}$, of the odd lorentzian is proportional to the projection of the spin in the QD along the measurement axis. The origin of the vertical offset, $y_{0}$, is unknown, but might be due to the broad KR feature from free electron spins in the quantum well, as discussed below. By repeating this measurement at various pump-probe delays, the evolution of the spin state can be mapped out.

When a magnetic field is applied along the $z$ axis, transverse to the injected spin (the Voigt geometry), the spin is quantized into eigenstates $|\uparrow\rangle$ and $|\downarrow\rangle$, with eigenvalues $S_{z}= \pm \hbar / 2$. The pump pulse initializes the spin at time $t=0$ into the superposition $|\psi(t=0)\rangle=(|\uparrow\rangle \pm|\downarrow\rangle) / \sqrt{2}$, for $\sigma^{ \pm}$polarized excitation. If isolated from its environment, the spin state then coherently evolves according to $|\psi(t)\rangle=\left(\mathrm{e}^{-i \Omega t / 2}|\uparrow\rangle \pm \mathrm{e}^{i \Omega t / 2}|\downarrow\rangle\right) / \sqrt{2}$, where $\hbar \Omega=g \mu_{\mathrm{B}} B_{z}$ is the Zeeman splitting. When the probe arrives at time $t=\Delta t$, the spin state is projected onto the $x$ axis, resulting in an average measured spin polarization of $\left\langle S_{x}(\Delta t)\right\rangle= \pm(\hbar / 2) \cos (\Omega \cdot \Delta t)$. This picture has not included the various environmental effects that cause spin decoherence and dephasing, inevitably leading to a reduction of the measured spin polarization with time.

The single-spin KR amplitude as a function of delay, measured with a 3-ns-duration probe pulse and a magnetic field $B=491 \mathrm{G}$, is shown in Fig. 2a, showing the expected oscillations due to the coherent evolution described above. Figure $2 \mathrm{~b}-\mathrm{f}$ shows a sequence of KR spectra at several delays, and the fits from which the data in Fig. 2a are obtained. In the inset of Fig. 2a the offset $y_{0}$ is shown, which oscillates with the same frequency as the single-spin KR but decays with a shorter lifetime. This behaviour may be consistent with that of free-electron spins in the quantum well, previously investigated in time-averaged measurements ${ }^{13}$.

In the simplest case, the evolution of the measured $\mathrm{KR}$ amplitude can be described by an exponentially decaying cosine,

$$
\theta(\Delta t)=A \cdot \Theta(\Delta t) \cdot \exp \left(\frac{-\Delta t}{T_{2}^{*}}\right) \cos (\Omega \cdot \Delta t),
$$

where $A$ is the overall amplitude, $\Theta(\Delta t)$ is the Heaviside step function and $T_{2}^{*}$ is the effective transverse spin lifetime (though this measurement eliminates ensemble averaging, the observed spin lifetime may be reduced from the transverse spin lifetime, $T_{2}$, by inhomogeneities that vary in time). To model our data, we sum over the contributions from each pump pulse separated by the repetition period $T_{\mathrm{r}}$, and convolve (denoted ' $*$ ') with the measured probepulse shape, $p(t)$,

$$
\theta_{0}(\Delta t)=p *\left[\sum_{n} \theta\left(\Delta t-n T_{\mathrm{r}}\right)\right] .
$$

The solid line in Fig. $2 \mathrm{a}$ is a fit to equation (2), yielding $\Omega=0.98 \pm 0.02 \mathrm{GHz}$ and $T_{2}^{*}=8.4 \pm 3.5 \mathrm{~ns}$. The dashed line shows equation (2) without the probe pulse convolution, plotted with the same parameters for comparison. 
a

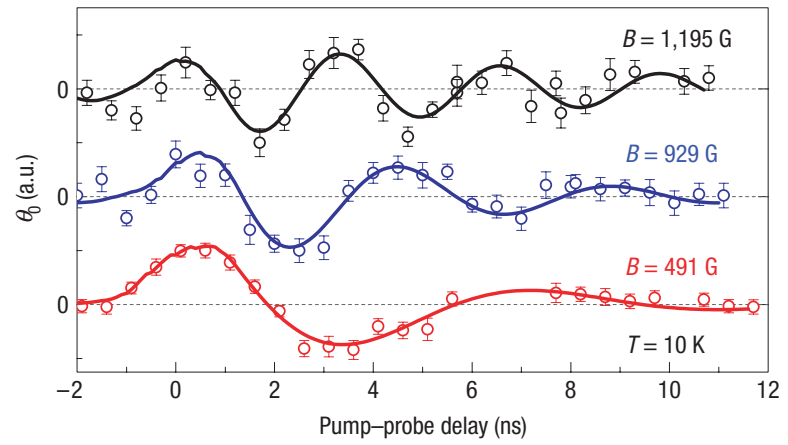

b
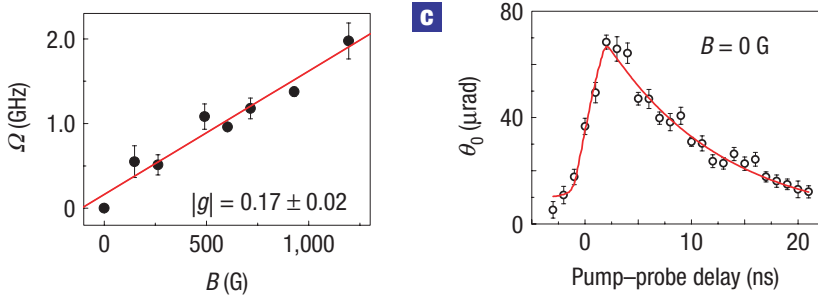

Figure 3 Magnetic-field dependence. a, Single-spin KR amplitude, $\theta_{0}$, as a function of delay at three different magnetic fields. The probe-pulse duration is 1.5, 1.5 and $2 \mathrm{~ns}$ from top to bottom. Solid lines are fits to equation (2) and the error bars indicate the standard error as obtained from the least-squares fit to the KR spectra. The data are vertically offset for clarity. $\mathbf{b}$, Precession frequency, $\Omega$, of the electron spin as a function of applied magnetic field, as obtained from fits to the data. Each data point is the average of several delay scans and the error bars indicate the root-mean-squared deviation of the measured frequencies. From a linear fit to the data (solid line) a $g$-factor of $0.17 \pm 0.02$ is obtained. c, $\theta_{0}$ as a function of delay at zero applied magnetic field, with $3 \mathrm{~ns}$ probe-pulse duration, and a fit to equation (2) yielding $T_{2}^{*}=10.9 \pm 0.5 \mathrm{~ns}$. Error bars indicate the standard error as obtained from the least-squares fit to the KR spectra.

In Fig. 3a the precession of the spin is shown at three different magnetic fields. As expected, the precession frequency increases with increasing field. The solid lines in Fig. $3 \mathrm{a}$ are fits to equation (2), and the frequency $\Omega$ obtained from such fits is shown in Fig. 3b as a function of magnetic field. A linear fit to these data yields an electron $g$-factor of $|g|=0.17 \pm 0.02$, consistent with the range of $g$-factors for these quantum dots found in previous ensemble or time-averaged measurements ${ }^{6,14}$. At zero magnetic field, as shown in Fig. 3c, the spin lifetime is found to be $T_{2}^{*}=10.9 \pm 0.5 \mathrm{~ns}$. This value agrees with previous time-averaged ${ }^{6}$ and ensemble ${ }^{13,14}$ measurements where the relevant decay mechanism is often suggested to be dephasing due to slow fluctuations in the nuclear spin polarization. However, these polarization fluctuations are not expected to result in a single-exponential decay of the electron $\operatorname{spin}^{15,16}$. Using these non-Markovian models to fit our data results in an increase of $\chi^{2}$ to 24.3 from 3.8 for the fit with a single-exponential decay, suggesting that other decay mechanisms than nuclear spin fluctuations might also be relevant in this case. In these QDs, the electronic level spacing of $\sim 1 \mathrm{meV}$ (ref. 17) is of the same order as $k_{\mathrm{B}} T$ for this temperature range. Therefore, thermally activated or phonon-mediated processes ${ }^{18-21}$ which yield an exponential decay might be significant in this regime.

This measurement technique is also sensitive to small nuclear spin polarizations. Ideally, there should be no induced steadystate nuclear polarization in this experimental geometry. Since the magnetic field is applied perpendicular to the direction of the spin, nuclear spins that are polarized by the electron spins
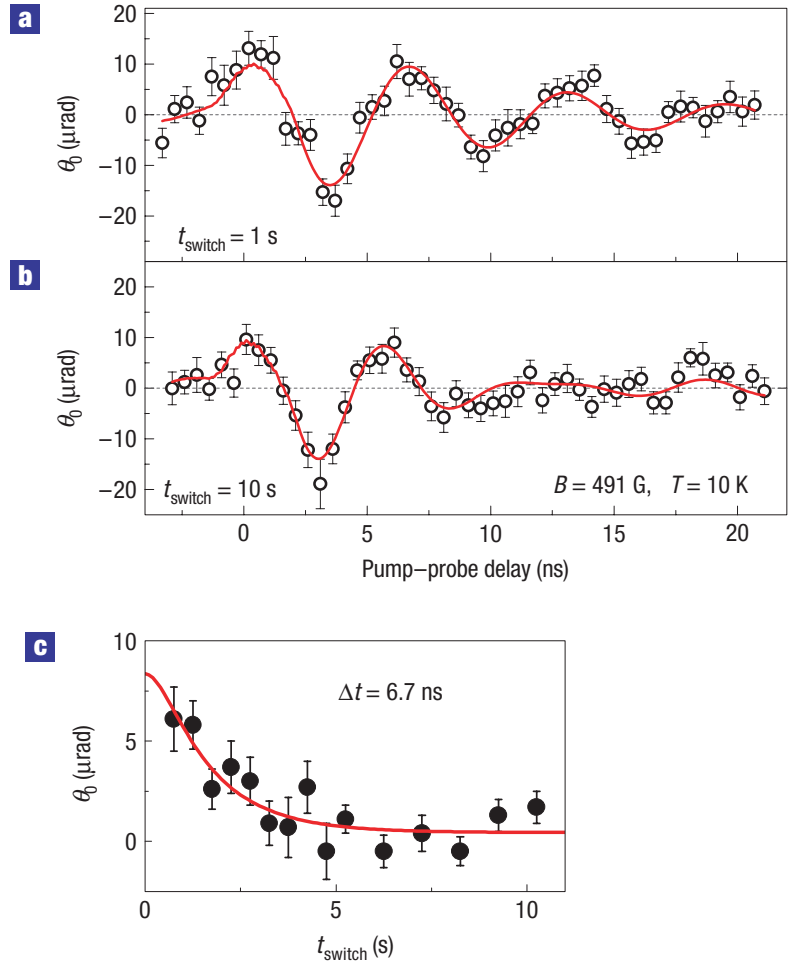

Figure 4 Probing the nuclear-spin environment. a,b, Single-spin KR amplitude, $\theta_{0}$, as a function of delay where the excitation helicity is switched between $\sigma^{+}$and $\sigma^{-}$polarization every $1 \mathrm{~s}\left(t_{\text {switch }}=1 \mathrm{~s}\right)(\mathbf{a})$ and every $10 \mathrm{~s}\left(t_{\text {switch }}=10 \mathrm{~s}\right)(\mathbf{b})$. The solid lines are fits to equation (3) convolved with the probe pulse, yielding a frequency shift due to the effective nuclear field of $\delta=0 \pm 0.04 \mathrm{GHz}$ for $t_{\text {switch }}=1 \mathrm{~s}$ and $\delta=0.14 \pm 0.02 \mathrm{GHz}$ for $t_{\text {switch }}=10 \mathrm{~s}$. Increased nuclear polarization is clearly seen as frequency beating when $t_{\text {switch }}=10 \mathrm{~s}$. c, KR amplitude as a function of $t_{\text {switch }}$ for a fixed pump-probe delay, $\Delta t$, of $6.7 \mathrm{~ns}$, revealing a DNP saturation time of $1.5 \mathrm{~s}$. Error bars indicate the standard error as obtained from the least-squares fit to the KR spectra.

precess around the applied field, resulting in zero steady-state polarization. However, for any misalignment of the pump laser from the perpendicular, there is a projection of the spin along the magnetic field, and right (left) circularly polarized light induces a small dynamic nuclear polarization (DNP) parallel (antiparallel) to the applied magnetic field ${ }^{11,22}$. Due to the hyperfine interaction this acts on the electron spin as an effective magnetic field, increasing (decreasing) the total magnetic field, resulting in a different precession frequency for right and left circularly polarized pump excitation. Since each data point is the difference of the KR signal with $\sigma^{+}$and $\sigma^{-}$polarized excitation, a small deviation from perpendicular between the magnetic field and the electron spin yields a measured KR signal

$$
\begin{aligned}
\theta(\Delta t)= & A \cdot \Theta(\Delta t) \cdot \exp \left(\frac{-\Delta t}{T_{2}^{*}}\right) \\
& \times[\cos ((\Omega+\delta) \Delta t)+\cos ((\Omega-\delta) \Delta t)],
\end{aligned}
$$

where $\delta=g \mu_{\mathrm{B}} \overline{B_{\text {nuc }}} / \hbar$ is the frequency shift due to the steady-state effective nuclear field, $\overline{B_{\text {nuc }}}$.

In order to investigate the effects of nuclear polarization on the electron spin, we have varied the rate $1 / t_{\text {switch }}$ at which the pump helicity is switched. Figure 4a, b shows the single-spin KR signal as a function of time with $t_{\text {switch }}=1 \mathrm{~s}$ (as in Fig. 2a), and $t_{\text {switch }}=10 \mathrm{~s}$, 
respectively, with otherwise identical conditions. With larger $t_{\text {switch }}$, the nuclear polarization has time to build, as is shown in Fig. $4 \mathrm{~b}$ by the visible beating, as expected from equation (3). A fit of the data in Fig. $4 \mathrm{a}\left(t_{\text {switch }}=1 \mathrm{~s}\right)$ to equation (3) convolved with the probe pulse yields $\delta=0 \pm 0.04 \mathrm{GHz}$ and $T_{2}^{*}=8.4 \mathrm{~ns}$, whereas for the data in Fig. $4 \mathrm{~b}\left(t_{\text {switch }}=10 \mathrm{~s}\right) \delta=0.14 \pm 0.02 \mathrm{GHz}$ and $T_{2}^{*}=8.3 \mathrm{~ns}$, with a $\chi^{2}$ of 2.3. For comparison, a single-frequency fit of the data in Fig. 4 b to equation (2) yields a slightly larger $\chi^{2}$ of 2.8, and a significantly shorter $T_{2}^{*}$ of $5.7 \mathrm{~ns}$. It is unlikely that $t_{\text {switch }}$ would have such an effect on the spin lifetime; moreover, nuclear polarization is the only effect in this system known to act on the spin with timescales on the order of seconds. Figure $4 \mathrm{c}$ shows the KR amplitude as a function of $t_{\text {switch }}$ for a fixed pump-probe delay. A fit of the data to equation (3) with $\delta$ saturating exponentially in $t_{\text {switch }}$ reveals a DNP saturation time of $1.5 \mathrm{~s}$, similar to what has previously been found in these $\mathrm{QDs}^{23}$. The nuclear polarization seen in Fig. 4b corresponds to an effective magnetic field of $100 \mathrm{G}$, or an electron spin splitting of $100 \mathrm{neV}$. For comparison, when the magnetic field and the electron spin are parallel, hyperfine spin splittings $\sim 1,000$ times larger have been observed ${ }^{23}$. Given the typical size of these QDs ${ }^{17}$, the electron interacts with $\sim 10^{5}$ nuclear spins. Since the hyperfine splitting here is smaller by a factor of 1,000 than the maximum observed splitting, the data shown in Fig. $4 \mathrm{~b}$ represent the detection of at most $\sim 10^{5} / 10^{3}=100$ polarized nuclear spins.

These measurements constitute a non-invasive optical probe of the coherent evolution of a single-electron spin state with nanosecond temporal resolution, which is a key ingredient for many spin/photon-based quantum-information proposals ${ }^{24,25}$. Furthermore, this technique provides a sensitive probe of the dynamics of the spin, revealing information about the spin coherence time and $g$-factor. Future work may exploit this ability to further explore the relevant decoherence mechanisms and the electron-nuclear spin interactions, and to observe the coherent manipulation of single spins in real time.

\section{METHODS}

A mode-locked Ti:sapphire laser provides pump pulses with energy $E_{\text {pump }}=1.653 \mathrm{eV}$ and duration $\sim 150 \mathrm{fs}$ at a repetition period $T_{\mathrm{r}}=13.1 \mathrm{~ns}$. The bandwidth of the spectrally broad pump pulses is narrowed to $\sim 1 \mathrm{meV}$ by passing the pump beam through a monochromator. The probe pulses are derived from a wavelength-tunable continuous-wave Ti:sapphire laser passing through an EOM, allowing for electrical control of the pulse duration from continuous wave down to $1.5 \mathrm{~ns}$. This technique yields short pulses while maintaining the narrow linewidth and wavelength tunability of the probe laser. Also, it enables us to adjust the pulse duration so as to maintain enough average power to achieve good signal-to-noise, while keeping the instantaneous power low enough to avoid unwanted nonlinear effects. The EOM is driven by an electrical pulse generator triggered by the pump laser, allowing for electrical control of the time delay between the pump and the probe pulses with picosecond precision. Additionally, in measurements with pump-probe delay $\Delta t>13 \mathrm{~ns}$ the pump beam has also been passed through an electro-optic pulse picker to increase the repetition period of the pulse train to $T_{\mathrm{r}}=26.2 \mathrm{~ns}$.

The sample is mounted on the cold finger of a He-flow cryostat at the focus of a microscope objective, at a temperature $T=10 \mathrm{~K}$. The pump and probe beams are focused and spatially overlapped on the sample with a spot size of $\sim 1 \mu \mathrm{m}$. The reflected light is collected through the same objective and the rotation of the probe polarization is detected by a balanced photodiode bridge. By chopping the pump beam at $4.1 \mathrm{kHz}$ and the probe beam at $20 \mathrm{~Hz}$, two lock-in amplifiers in series serve to isolate the KR signal induced by the pump. The pump polarization is switched between $\sigma^{+}$and $\sigma^{-}$with a liquid crystal retarder at a rate $1 / t_{\text {switch }}$, and a measurement of the rotation angle is taken at each helicity, with several seconds of averaging for noise reduction (that is, the spin is initialized and probed repeatedly for each measurement). The spin-dependent signal is then obtained by subtracting the measurements at each helicity. Typical time-averaged pump and probe intensities incident on the sample are $20 \mathrm{~W} \mathrm{~cm}^{-2}$ and $200 \mathrm{~W} \mathrm{~cm}^{-2}$, respectively.

The sample is grown by molecular beam epitaxy and consists of a single $4.2 \mathrm{~nm}$ GaAs quantum well in the centre of a planar $\mathrm{Al}_{0.3} \mathrm{Ga}_{0.7}$ As $\lambda$-cavity. A 2 min growth interruption at each quantum well interface enables large $\left(\sim 100\right.$-nm-diameter $\left.{ }^{17}\right)$ monolayer thickness fluctuations to develop that act as $\mathrm{QDs}^{26,27}$. The front and back cavity mirrors are distributed Bragg reflectors composed of five and 28 pairs of $\mathrm{AlAs} / \mathrm{Al}_{0.3} \mathrm{Ga}_{0.7} \mathrm{As} \lambda / 4$ layers, respectively. This asymmetrical design enables light to be injected into and emitted from the cavity on the same side. The cavity resonance is centred at $1.624 \mathrm{eV}$ at $10 \mathrm{~K}$ and has a quality factor of 120 . We expect the KR at the peak of the resonance to be enhanced by a factor of $\sim 15$ (refs 28,29$)$.

\section{Received 29 May 2007; accepted 6 September 2007; published 7 October 2007.}

\section{References}

1. Loss, D. \& DiVincenzo, D. P. Quantum computation with quantum dots. Phys. Rev. A 57, 120-126 (1998).

2. Awschalom, D. D., Loss, D. \& Samarth, N. (eds) Semiconductor Spintronics and Quantum Computation (Springer, Berlin, 2002).

3. Hanson, R., Kouwenhoven, L. P., Petta, J. R., Tarucha, S. \& Vandersypen, L. M. K. Spins in few-electron quantum dots. Rev. Mod. Phys. (in the press); preprint at $<$ http://arxiv.org/abs/cond-mat/0610433> (2006).

4. Berezovsky, J. et al. Nondestructive optical measurements of a single electron spin in a quantum dot. Science 314, 1916-1920 (2006).

5. Atatüre, M., Dreiser, J., Badolato, A. \& Imamoglu, A. Observation of Faraday rotation from a single confined spin. Nature Phys. 3, 101-105 (2007).

6. Bracker, A. S. et al. Optical pumping of the electronic and nuclear spin of single charge-tunable quantum dots. Phys. Rev. Lett. 94, 047402 (2005).

7. Ebbens, A. et al. Optical orientation and control of spin memory in individual InGaAs quantum dots. Phys. Rev. B 72, 073307 (2005).

8. Högele, A. et al. Spin-selective optical absorption of singly charged excitons in a quantum dot. Appl. Phys. Lett. 86, 221905 (2005).

9. Meier, F. \& Awschalom, D. D. Faraday rotation spectroscopy of quantum-dot quantum wells. Phys. Rev. B 71, 205315 (2005).

10. Warburton, R. J. et al. Optical emission from a charge-tunable quantum ring. Nature 405 926-929 (2000).

11. Meier, F. \& Zakharchenya, B. P. (eds) Optical Orientation: Modern Problems in Condensed Matter Sciences (North Holland, Amsterdam, 1984).

12. Bracker, A. S. et al. Binding energies of positive and negative trions: From quantum wells to quantum dots. Phys. Rev. B 72, 035332 (2005).

13. Dzhioev, R. I. et al. Optical orientation and the Hanle effect of neutral and negatively charged excitons in $\mathrm{GaAs} / \mathrm{Al}_{x} \mathrm{Ga}_{1-x}$ As quantum wells. Phys. Rev. B 66, 153409 (2002).

14. Dutt, M. V. G. et al. Stimulated and spontaneous optical generation of electron spin coherence in charged GaAs quantum dots. Phys. Rev. Lett. 94, 227403 (2005).

15. Khaetskii, A. V., Loss, D. \& Glazman, L. Electron spin decoherence in quantum dots due to interaction with nuclei. Phys. Rev. Lett. 88, 186802 (2002).

16. Merkulov, I. A., Efros, Al. L. \& Rosen, M. Electron spin relaxation by nuclei in semiconductor quantum dots. Phys. Rev. B 65, 205309 (2002).

17. Gammon, D., Snow, E. S., Shanabrook, B. V., Katzer, D. S. \& Park, D. Fine structure splitting in the optical spectra of single GaAs quantum dots. Phys. Rev. Lett. 76, 3005-3008 (1996).

18. Semenov, Y. G. \& Kim, K. W. Phonon-mediated electron-spin phase diffusion in a quantum dot. Phys. Rev. Lett. 92, 026601 (2004).

19. Golovach, V. N., Khaetskii, A. \& Loss, D. Phonon-induced decay of the electron spin in quantum dots. Phys. Rev. Lett. 93, 016601 (2004).

20. Khaetskii, A. V. \& Nazarov, Y. V. Spin-flip transitions between Zeeman sublevels in semiconductor quantum dots. Phys. Rev. B 64, 125316 (2001).

21. Erlingsson, S. I., Nazarov, Y. V. \& Fal'ko, V. I. Nucleus-mediated spin-flip transitions in GaAs quantum dots. Phys. Rev. B 64, 195306 (2001).

22. Salis, G., Awschalom, D. D., Ohno, Y. \& Ohno, H. Origin of enhanced dynamic nuclear polarization and all-optical nuclear magnetic resonance in GaAs quantum wells. Phys. Rev. B 64, 195304 (2001).

23. Gammon, D. et al. Electron and nuclear spin interactions in the optical spectra of single GaAs quantum dots. Phys. Rev. Lett. 86, 5176-5179 (2001).

24. Meier, F. \& Awschalom, D. D. Spin-photon dynamics of quantum dots in two-mode cavities. Phys Rev. B 70, 205329 (2004).

25. Leuenberger, M. N. Fault-tolerant quantum computing with coded spins using the conditional Faraday rotation in quantum dots. Phys. Rev. B 73, 075312 (2006).

26. Zrenner, A. et al. Quantum dots formed by interface fluctuations in a AlAs/GaAs coupled quantum well structures. Phys. Rev. Lett. 72, 3382 (1994).

27. Gammon, D., Snow, E. S., Shanabrook, B. V., Katzer, D. S. \& Park, D. Homogeneous linewidths in the optical spectrum of a single gallium arsenide quantum dot. Science 273, 87-90 (1996).

28. Salis, G. \& Moser, M. Faraday-rotation spectrum of electron spins in a microcavity-embedded GaAs quantum wells. Phys. Rev. B 72, 115325 (2005).

29. Li, Y. Q. et al. Cavity enhanced Faraday rotation of semiconductor quantum dots. Appl. Phys. Lett. 88, 193126 (2006).

\section{Acknowledgements}

We acknowledge support from NSF and the Air Force Office of Scientific Research.

Correspondence and requests for materials should be addressed to D.D.A.

Reprints and permission information is available online at http://npg.nature.com/reprintsandpermissions/ 